\title{
Surgical treatment of emphysema
}

Tratamento cirúrgico do enfisema

\section{José de Jesus Peixoto Camargo}

Smoking was undoubtedly the worst illness of the 20th century. As its most tragic legacy, it has left us with pulmonary emphysema. Worldwide, pulmonary emphysema is responsible for 2.74 million deaths annually, according to a report issued by the World Health Organization in 2000.(1)

In emphysema, as in most degenerative diseases, the treatment is typically palliative, aimed at improving patient quality of life, without, however, having any substantial effect on the natural course of the illness.

In cases of bullous emphysema, surgical treatment is the obvious choice, assuming that the patient meets the functional selection criteria. ${ }^{(2)}$ Although the same can be said for diffuse emphysema, the surgical techniques employed to treat the latter have been some of the most varied and challenging ever attempted.

The most devastating consequences of pulmonary emphysema are hyperinflation and air trapping.

The increase in total lung capacity impairs the chest-wall mechanics in that the thorax reaches the limit of expansion.

The trapped air approximates the residual volume of the total lung capacity, thereby reducing slow vital capacity, as well as FVC and FEV .

Smaller differences between residual volume and FVC translate to greater respiratory effort, and, with the progression of the hyperinflation, there is a gradual decrease in ventilatory capacity, leading patients to experience respiratory difficulty, even at rest.

It is therefore easy to surmise that the principal objective in the treatment of emphysema is to reduce the volume of trapped air.

Based on the concept that, in emphysema, lung volume increases considerably, filling the rib cage to capacity, some surgical procedures have been devised in order to increase the size of the rib cage and to allow further increases in lung volume.

Among such surgical procedures are costochondrectomy, proposed by Freund in 1906, and paravertebral thoracoplasty, devised by Voelcker in $1926,{ }^{(3)}$ both of which had the objective of minimizing the rigidity of rib cage and allowing better accommodation of hyperinflated emphysematous lungs.

Although satisfactory initial results were described in up to $83 \%$ of cases, the benefit of these techniques was fleeting, since the symptoms returned after the skeleton consolidated, reestablishing the rigidity of the chest wall.

Other attempts were aimed at reducing the size of the lungs, using maneuvers to elevate the diaphragm, either by external compression (pneumoperitoneum and abdominal belts) or by elevation of the diaphragm through denervation (phrenicectomy).

The inconsistent and transitory nature of the results relegated these techniques to ostracism.

\section{Lung volume reduction surgery}

It was in the 1950s when a group of authors first hypothesized that the distention of the rib cage observed in patients with emphysema plays an important role in the dyspnea observed in such cases. ${ }^{(4)}$ The authors postulated that a decrease in the size of the lungs, with consequent recovery of the thoracic bellows, could play a role in the treatment of select cases.

The selection would be made based on a finding of emphysema with heterogeneous distribution, with areas relatively preserved.

The surgical excision of the areas most affected would reduce lung volume, allowing the diaphragm and the chest wall to return to their anatomical positions, together with recovery of the thoracic bellows.

With the reduction in lung volume, there would also be decompression of potentially functional areas.

This proposal is, therefore, based on surgical correction of refractory lung hyperinflation, a condition widely recognized as being untreatable at the clinical level. ${ }^{(4)}$

These ideas were tested by the authors, who obtained favorable functional results. However, 
due to the poor technical conditions at the time, mortality was high.

Surgery for emphysema never lost its popularity in Europe and South America. ${ }^{(5)}$ However, there was an extraordinary expansion of its use worldwide after one group of authors ${ }^{(6)}$ proposed the adoption of more well-defined criteria for the selection of patients, giving greater weight to pre-operative rehabilitation, as well as introducing the use of mechanical sutures protected with bovine pericardium in order to reduce air leaks, together with an infrastructure that involves anesthesiologists and intensive care professionals familiarized with the treatment of lung transplant patients.

Those authors published their initial experience with 20 patients, among whom there were no deaths and the degree of morbidity was acceptable.

The excessive enthusiasm and disregard for the selection criteria produced catastrophic results at facilities that were unprepared to perform surgical procedures of high complexity.

The inconsistent benefits and the high costs led the sources of funding for health care in the USA to implement a new protocol, the National Emphysema Treatment Trial (NETT), charged with comparing, in a randomized manner, the yield of lung volume reduction surgery with that of the best possible clinical treatment, included within which is pulmonary rehabilitation. ${ }^{(7)}$

All of the patients were started on a program of pulmonary and nutritional rehabilitation. After 2 months, the patients were randomly divided into two groups: those that were referred for surgical treatment (with the use of video-assisted thoracoscopy or an open technique); and those that continued the clinical treatment.

The following criteria were established in order to determine the eligibility of the candidates:

1)Indication criteria: (i) dyspnea caused by emphysema (and not by bronchitis or asthma) despite appropriate clinical treatment; (ii) age < 75 years; (iii) abstinence from smoking $>4$ months; (iv) postbronchodilator $\mathrm{FEV}_{1}<35-40 \%$ of predicted; (v) total lung capacity $>120 \%$ of predicted; (vi) residual volume $>175 \%$ of predicted; and (vii) sever hyperinflation preferentially affecting the upper lobes.

2) Contraindication criteria: (i) significant comorbidities; (ii) moderate to severe bronchitis; (iii) alpha-1 antitrypsin deficiency; (iv) obesity or wasting; (v) postbronchodilator FEV $<20 \%$ of predicted. (v) kyphoscoliosis; (vi) mean pulmonary artery pressure $>35 \mathrm{mmHg}$; (vii) continued smoking; (viii) $\mathrm{PaCO}_{2}>55 \mathrm{mmHg}$; (ix) life expectancy < 2 years; ( $x$ ) DLCO $<20 \%$; (xi) ccompanying coronary disease; (xii) pleural symphysis; and (xiii) homogeneous disease.

3)An ideal candidate for lung volume reduction surgery presents the following: less than 70 years of age; severe hyperinflation, without a significant bronchial component; no major comorbidities; heterogeneous emphysema, predominantly in the upper lobes; and low initial exercise capacity.

While the protocol was still underway, an extemporaneous paper, which was circulating on the Internet many months before its official publication, ${ }^{(8)}$ called attention to the functional criteria for identifying patients at high risk: FEV $_{1}<20 \%$ of predicted; DLCO $<20 \%$; and homogeneous disease.

The paper called attention to the fact that the presence of any one of these three elements would lead to death within the first 30 postoperative days in $16 \%$ of the population undergoing the surgical procedure, compared with $0 \%$ of the population receiving clinical treatment only.

When all three elements were present, mortality reached $25 \%$.

These observations were released to the lay press, evidently due to pressure from health insurance providers attempting to justify denial of payment for the procedure.

The resulting dispute called attention to the fact that the more important question was not the focus of the press release. ${ }^{(9)}$

Surgery would not have been indicated for such patients, because they would not have presented the eligibility criteria established by the NETT.

A total of 1,218 patients were randomized after having met the prerequisite of pulmonary rehabilitation.

The patients were then referred for surgical treatment (sternotomy or video-assisted surgery) or for continuation of the best possible clinical treatment.

A summary of the results obtained is shown in Table 1.

The best patients were in group 1, characterized by emphysema predominantly in the upper 
Table 1 - Results of the National Emphysema Treatment Tria ${ }^{99}$ in relation to the groups submitted to clinical or surgical treatment.

\begin{tabular}{lcccc}
\hline \multicolumn{1}{c}{ Characteristic } & Group 1 & Group 2 & Group 3 & Group 4 \\
\hline Type of disease & Apical & Apical & Basal & Basal \\
Exercise tolerance & Low & High & Low & High \\
Mortality & Lower in the & No difference & No difference & Higher in the \\
& surgery group & between groups & between groups & surgery group \\
Physical performance & Better in the & Better in the & No difference & No difference \\
at 24 months & surgery group & surgery group & between groups & between groups \\
Quality of life & Better in the & Better in the & Better in the & No difference \\
& surgery group & surgery group & surgery group & between groups \\
\hline
\end{tabular}

lobes and low initial exercise capacity, whereas the worst were in group 4, characterized by emphysema predominantly in the lung bases and high initial exercise capacity.

Based on the functional gains and the duration of the benefit, it was established that the health insurance providers would approve the procedure for patients in group 1, would definitively disapprove the procedure for those in group 4 and would approve or disapprove the procedure on a case-by-case basis for those in groups 2 and 3 .

The conclusions of the NETT were as follows: (i) in the overall evaluation, life expectancy is greater in the population undergoing the surgical procedure, despite the fact that the numbers have yet to reach statistical significance; (ii) exercise capacity and quality of life are better in the population undergoing the surgical procedure; (iii) the best candidates for lung volume reduction surgery are those with an apical presentation and low initial exercise capacity; and (iv) open surgery and video-assisted surgery provide identical results.

\section{Lung transplantation in emphysema}

Emphysema is the leading indication for lung transplant, patients with emphysema accounting for approximately 55\% of all cases.

The selection criteria are rigid, and the results obtained present a quite direct relationship with these criteria.

General indication for lung transplantation: terminal lung disease, with severe functional limitation; life expectancy of less than 2 years; no other treatment options; no comorbidities; less than 65 years of age; appropriate psychosocial and family profile; and a strong will to live.
Outpatients are preferred, transplantation rarely being recommended for critically ill patients..$^{(10)}$

Specific indication in emphysema: a spirometry finding of FEV below 20\% of predicted is usually indicative of a life expectancy of less than 18 months.

However, among patients presenting a $\mathrm{PaCO}_{2}>51 \mathrm{mmHg}$, the two-year survival rate is only $50 \%$.

We should also consider, for all indications, the so-called window of opportunity, since the waiting list is growing due to the limited availability of organs. Therefore, it is possible that transplants will be postponed, and emergency transplants are impractical.

The following criteria are applied for the inclusion of patients on the waiting list: (i) postbronchodilator $\mathrm{FEV}_{1}<25 \%$ of predicted; (ii) DLCO $<20 \%$ of predicted; (iii) resting hypoxemia $\left(\mathrm{PaO}_{2}<55-60 \mathrm{mmHg}\right)$; (iii) hypercapnia $\left(\mathrm{PaCO}_{2}>50 \mathrm{mmHg}\right)$; (iv) significant secondary pulmonary hypertension (mean pulmonary artery pressure $>35 \mathrm{mmHg}$ or systolic pulmonary artery pressure > $45 \mathrm{mmHg}$ ); (v) significant drop in $\mathrm{FEV}_{1}$; (vi) frequent hospitalizations, suggesting a lack of control of the disease; (vii) (preferentially) dependent on oxygen therapy; and (viii) more recently, grade IV dyspnea, non-candidacy for lung volume reduction surgery, homogeneous disease, alpha- 1 antitrypsin deficiency, and a body mass index $<20 \mathrm{~kg} / \mathrm{m}^{2}$ have been identified as elements that should make inclusion on the COPD waiting list more urgent.

Technically, the obvious tendency is to transplant the most affected lung.

This selection is made based on the perfusion scintigraphy findings.

When the illness is symmetrical, transplant of the right lung is preferable. 
Lung transplantation for emphysema can be unilateral or bilateral. However, in recent years, double-lung transplant has been indicated more frequently, especially in the population of patients below the age of 50 (among which are nearly all of the patients with alpha-1 antitrypsin deficiency).

This increase occurred after it was demonstrated that the functional results and the five-year survival rate are better in the population submitted to bilateral transplant (66.7\% vs. 44.9\%). ${ }^{(11)}$

The comparative analysis among patients of similar profiles on waiting lists demonstrate that, although transplant has um indisputable impact on the quality of life of transplant recipients, it does not significantly alter the five-year survival rate in this population, which hovers around 55\%.

One of the arguments cited for this loss of statistical significance in comparison with the population receiving clinical treatment only is that, since the wait for a lung transplant in the USA is quite long (more than 2 years), physicians put their patients on waiting lists earlier, in the hope that, when the critical moment finally arrives, their patients will be near the top of the list.

This practice would increase the survival of the population on the waiting list and induce an error in the comparative analysis of the two groups, reducing the apparent benefit in terms of survival of the transplant population.

However, the potential for morbidity among transplant recipients is considerable due to the inevitability of immunosuppression, and at least $30 \%$ are readmitted to the hospital.

The cost of a lung transplant, which is considered an expensive procedure in Brazil, is still less than that of the maintenance treatment of a patient with severe emphysema, especially if the need for frequent hospitalizations and continuous oxygen therapy are factored in.

If the rigid selection criteria are applied, no more than $10 \%$ of the patients with severe emphysema will effectively be considered candidates for lung transplantation.

\section{Recent advances in the surgical treatment of emphysema}

Weighing the pros and cons of classic lung volume reduction surgery and lung transplantation, taking into consideration the fact that, together, these procedures benefit no more than $20 \%$ of critically ill patients, other treatment options that reduce lung hyperinflation have come to be proposed.
Recently, the ideas of one group of authors ${ }^{(12)}$ were expanded upon in the study by Saad et al., ${ }^{(13)}$ published in the current issue of the Brazilian Journal of Pulmonology. Saad et al. proposed external drainage of diffuse emphysema, similar to that reported in another study, ${ }^{(14)}$ for the treatment of patients with bullous emphysema who present poor function, precluding the use of conventional bullectomy.

Saad et al. ${ }^{(13)}$ proposed drainage using a silicone chest tube, inserted under local anesthesia through the chest wall into the emphysematous parenchyma.

Their report of three cases describes favorable medium-term functional evolution.

The proposal is to maintain the chest tube indefinitely, naturally with the inconveniences of having an open wound and the permanent escape of air through the drain.

As disagreeable as the initial impact of this proposal might be, we must consider the scarcity of alternatives and the acceptance of the treatment, probably celebrated by these patients, whose idea of quality of life had been subverted by their chronic suffering.

In recent years, techniques of endoscopic volume have been proposed. Although most are still in the experimental phase, they have undeniable potential for clinical use.

Three different techniques have been proposed: a) fenestration of the bronchial wall and the placement of stents that facilitate the drainage of the most emphysematous areas; b) implantation of unidirectional bronchial valves with the intention of promoting atelectasis and blocking the inspiratory flow; and c) instillation of biological glue with the objective of provoking the collapse and remodeling of the emphysematous areas.

\section{Fenestration of the bronchial wall}

The creation of communication between the most emphysematous zones and the skin of the patient, simulating the spiracles in the bodies of insects, was first described in 1978 by Macklem. ${ }^{(12)}$ The authors of a study conducted in 2002 investigated the use of this technique, which, through the placement of stents in the bronchial wall, creates extra-anatomical shortcircuits, establishing communication between the lung parenchyma, distended by collateral ventilation, and the largest airway. ${ }^{(15)}$ 
As a consequence, there is a reduction in the volume of the emphysematous parenchyma affected by this new passage for the outflow of air.

Although the method is technically simple, it requires the use of Doppler echocardiogram via endoscopy in order to identify the "mute" areas of the bronchial wall (areas that are not in contact with the adjacent pulmonary vessels.

After these areas have been identified, the cartilage is perforated, allowing the introduction of the small spreaders and the insertion of the stents, with the intention of maintaining the patency of these passages.

All of this instrumentation is introduced through the working channel of the fiberoptic bronchoscope.

The study involved the use of stents covered with substances that prevent or delay the formation of granulomas, which was expected to present a great obstacle to maintaining the system patent and functional.

One study specifically compared the use of pure silicone stents and paclitaxel-eluting stents. ${ }^{(16)}$ The authors demonstrated that the antiblastic effect retarded the formation of granulomas, maintaining patency for a longer time. ${ }^{(16)}$

The functional gain is evident in the first month, and the mean reduction in residual volume is $400 \mathrm{~mL}$ after 6 months, which explains the reduction observed in the degree dyspnea during this same period.

ln a study of 36 patients treated at 7 different facilities, one death occurred, due to intraoperative bleeding. In 2 other patients, due to technical difficulties, the stents could not be placed.

The most common complication was worsening of the respiratory infection..$^{(17)}$

The most critical question related to the method is the tendency for occlusion of the stents by granulation tissue, a complication that was perfectly predictable if we consider that a foreign body is placed in the wall of the airway, where there is secretion and bacterial colonization.

In early 2009, the results of the protocol carried out at 7 international centers will be submitted to critical evaluation, in order to determine whether it will be used in routine clinical practice.

\section{Bronchial valves}

Patients with severe emphysema and predominance of the disease in the upper lobes, without other comorbidities or significant bronchial disease, can be treated through the endoscopic implantation of unidirectional bronchial valves, with the intention of promoting a reduction in lung volume.

In a study involving 19 patients, the valves were placed unilaterally in 8 patients and bilaterally in 11.

The trend is to occlude the three segmental bronchi of the upper lobe selected.

In the majority of cases, the valves were well tolerated.

One patient developed pneumothorax, and the valve placed in one segmental bronchus of the upper lobe was therefore removed, resulting in spontaneous, favorable evolution of the complication.

Two other patients presented, respectively, a significant increase in bronchial secretion and severe bronchospasm.

In both cases, the valves were removed after a few days, with clinical monitoring of the situation.

An alteration of $\geq 4 \%$ following the procedure translates to a significant improvement in quality of life, according to the specific protocol for pulmonary evaluation. ${ }^{(18)}$

In a study using the index known as BODE (Body mass index, airway Obstruction, Dyspnea, and Exercise capacity) and the Saint George's Respiratory Questionnaire, No statistical improvement was observed in pulmonary function at 12 or 24 months, although there was a sustained increase in $\mathrm{FEV}_{1}(>12 \%)$ in 1 of the 5 patients and in FVC $(>150 \mathrm{~mL})$ in 3 of the 5 patients at 24 months. ${ }^{(19)}$

The principal complication observed was the formation of small granulomas around the valve, albeit without functional impairment of the valve.

With the use of valves that are more modern and have a more favorable design, reducing the area of traumatic contact with the bronchial wall, the occurrence of such granulomas has decreased dramatically.

The conclusion of the authors is that endoscopic treatment of emphysema can be conducted in a safe and easily reversible manner, and their final suggestion is that further randomized studies be carried out in order to determine with precision the benefits of such treatment in the management of this progressive disease. 


\section{Instillation of biological glue}

The instillation of biological glue via endoscopy, with the intention of occluding the bronchi and consequently reducing the volume of the pulmonary segments ventilated by the occluded bronchi, has been offered up as an alternative that is less costly and technically less complex than are those previously described. ${ }^{(20)}$

The problems that can be expected are basically the same as those expected with the other techniques of endobronchial management, and the long-term results remain unknown.

\section{Where do we go from here?}

Abstinence from smoking, oxygen therapy and traditional lung volume reduction surgery are the only strategies that have proven to be effective in increasing the survival of patients with severe emphysema.

With endoscopic techniques, despite the industry-promoted appeal from the media, the initial results are discouraging in terms of the duration of the benefits, especially if compared with those of lung volume reduction surgery, as well as in terms of the functional gains, which are minimal in comparison with those obtained after lung transplantation.

However, taking into consideration the complexity of and the morbidity resulting from traditional methods, as well as, especially, the rigidity of the selection criteria, which limits the use of either method considerably, we should undoubtedly encourage research into new and more effective forms of treatment aimed at minimizing the suffering of these patients.

\section{José de Jesus Peixoto Camargo}

Professor Adjunto de Cirurgia Torácica da Faculdade Federal de Ciências Médicas de Porto Alegre, Porto Alegre, Rio Grande do Sul

\section{References}

1. Burney P, Suissa S, Soriano JB, Vollmer WM, Viegi G, Sullivan SD, et al. The pharmacoepidemiology of COPD: recent advances and methodological discussion. Eur Respir J Suppl. 2003;43:1s-44s.

2. Snider G. Reduction pneumoplasty for giant bullous emphysema. Implications for surgical treatment of nonbullous emphysema. Chest. 1996;109(2):540-8. Review.

3. Deslauriers J. A perspective on the role of surgery in chronic obstructive lung disease. Chest Surg Clin N Am. 1995;5(4):575-602.

4. Brantigan OC, Mueller E. Surgical treatment of pulmonary emphysema. Am Surg. 1957;23(9):789-804.

5. Crosa-Dorado V, Pomi J, Perez-Penco E, Carriquiry G. Treatment of dyspnea in emphysema: Pulmonary remodeling; hemo- and pneumostatic suturing of the emphysematous lung. Res Surg 1992;4:152-155.

6. Cooper JD, Patterson GA. Lung-volume reduction surgery for severe emphysema. Chest Surg Clin N Am. 1995;5(4):815-31.

7. Fishman A, Martinez F, Naunheim K, Piantadosi S, Wise $\mathrm{R}$, Ries $\mathrm{A}$, et al. A randomized trial comparing lungvolume-reduction surgery with medical therapy for severe emphysema. N Engl J Med. 2003;348(21):2059-73.

8. National Emphysema Treatment Trial Research Group. Patients at high risk of death after lung-volume-reduction surgery. N Engl J Med. 2001;345(15):1075-83.

9. Cooper JD. Paying the piper: the NETT strikes a sour note. National Emphysema Treatment Trial. Ann Thorac Surg. 2001;72(2):330-3.

10. Meyers BF, Yusen RD, Lefrak SS, Cooper JD. Improved longterm survival seen after lung volume reduction surgery compared to continued medical therapy for emphysema. Ann Thorac Surg. 2001;71(6):2081.

11. Cassivi SD, Meyers BF, Battafarano RJ, Guthrie TJ, Trulock EP, Lynch JP, et al. Thirteen-year experience in lung transplantation for emphysema. Ann Thorac Surg. 2002;74(5):1663-9.

12. Macklem, PT. Collateral ventilation. N Engl J Med. 1978;298(1):49-50.

13. Saad R, Neto VD, Botter M, Stirbulov R, Rivaben JH, Gonçalves R. Therapeutic application of collateral ventilation with pulmonary drainage in the treatment of diffuse emphysema: report of the first three cases. J Bras Pneumol. 2009;35(1): XXX-XXX.

14. Venn GE, Williams PR, Goldstraw P. Intracavity drainage for bullous, emphysematous lung disease: experience with the Brompton technique. Thorax. 1988;43(12):998-1002.

15. Cooper JD. Clinical trials and future prospects for lung volume reduction surgery. Semin Thorac Cardiovasc Surg. 2002;14(4):365-70.

16. Choong CK, Phan L, Massetti P, Haddad FJ, Martinez C, Roschak E, et al. Prolongation of patency of airway bypass stents with use of drug-eluting stents. J Thorac Cardiovasc Surg. 2006;131(1):60-4.

17. Cardoso PF, Snell Gl, Hopkins P, Sybrecht GW, Stamatis $\mathrm{G}, \mathrm{Ng} \mathrm{AW}$, et al. Clinical application of airway bypass with paclitaxel-eluting stents: early results. J Thorac Cardiovasc Surg. 2007;134(4):974-81.

18. Jones PW, Quirk FH, Baveystock CM. The St George's Respiratory Questionnaire. Respir Med. 1991;85 Suppl B:25-31; discussion 33-7.

19. de Oliveira HG, Macedo-Neto AV, John AB, Jungblut S, Prolla JC, Menna-Barreto SS, et al. Transbronchoscopic pulmonary emphysema treatment: 1-month to 24-month endoscopic follow-up. Chest. 2006;130(1):190-9.

20. Ingenito EP, Wood DE, Utz JP. Bronchoscopic lung volume reduction in severe emphysema. Proc Am Thorac Soc. 2008;5(4):454-60. 